For numbered affiliations see end of article.

monique.andersson@ouh.nhs.uk Cite this as: BMJ 2020;369:m2420 http://dx.doi.org/10.1136/bmj.m2420 Published: 24 June 2020
COVID 19: ROLL OUT OF ANTIBODY TESTING

\section{Rapid roll out of SARS-CoV-2 antibody testing-a concern}

Monique Andersson, ${ }^{1}$ Nicola Low, ${ }^{2}$ Neil French, ${ }^{3}$ Trisha Greenhalgh, ${ }^{4}$ Katie Jeffery, ${ }^{5}$ Andrew Brent, ${ }^{5}$ Jonathan Ball, ${ }^{6}$ Allyson Pollock, ${ }^{7}$ David McCoy, ${ }^{8}$ Miren Iturriza-Gomara, ${ }^{9}$ Iain Buchan, ${ }^{10}$ Helen Salisbury, ${ }^{4}$ Deenan Pillay, ${ }^{11}$ Will Irving ${ }^{12}$

We are writing to express concerns over aspects of the establishment of SARS-CoV-2 antibody testing in England. NHS England and NHS Improvement wrote to NHS trusts and pathology networks on 25 May 2020, asking them to offer antibody testing at short notice and ramp up capacity to thousands of samples a day. ${ }^{12}$

We have three concerns about the request. Firstly, there is no specific clinical indication for the test on an individual basis. Secondly, the performance of these assays has not yet been assessed to the standard typically required of a novel test. And thirdly, the resource implications are not considered.

We support the rapid provision of diagnostic tests for individual and public health and recognise the need to deliver at pace. It is essential, however, that quality systems, which have evolved over many years and are the foundation for delivering the right result of the right test to the right person at the right time, are not circumvented.

\section{No clinical indication}

In usual clinical practice, antibody testing fulfils several purposes. In acute illness, an IgM antibody response can be used to diagnose an infectious cause. The presence of IgG antibodies can provide evidence of prior infection or vaccination and likely immunity to future infection with the same agent for some viruses, such as measles or hepatitis A viruses. For other viruses (including hepatitis $C$ virus, influenza virus, respiratory syncytial virus) IgG antibodies do not guarantee immunity, but because they indicate prior exposure (infection), population testing might yield useful information about previous spread of infection.

The SARS-CoV-2 antibody tests being rolled out detect IgG class or "total" antibodies and do not fulfil these first two indications. They are not diagnostic tests, for which genome detection assays are used. And because the test has been made available to all patients who would like a test, rather than for a specific clinical indication, neither a positive nor negative result is likely to alter clinical management, and a positive result does not indicate immunity.

The concept of "immune passports," allowing healthcare workers or others to work, has not been established. Those with a positive antibody test should still consider themselves at risk and follow infection control policies designed to prevent nosocomial spread and risk of infection. There is, therefore, no benefit to healthcare organisations or to others in knowing the status of employees at present.

\section{Unproved performance}

No reference standard has been defined for SARS-CoV-2 antibody tests. Access to "true positive" and "true negative" samples is difficult, even in large teaching hospitals. Laboratories across the country will have found it hard to achieve the necessary standard verification in a matter of days, not least because different platforms will be used for this test from manufacturers including Abbott, Roche, Siemens, Ortho, and Diasorin. Currently, there are no openly available data to compare the performance of these platforms. Public Health England has published verification data, but concerns remain about the breadth of that verification. Those who are at highest risk of death from this infection are elderly people, those from black and minority ethnic groups, and immunocompromised people. There are currently no data showing the performance of the tests in these groups. The correct route to generating valid test performance data is well designed prospective clinical studies. The assay is being rolled out at an unprecedented pace and scale without adequate assessment, potentially compromising public trust in pathology services in the future.

\section{Wasted resources}

NHS England requires the result to be available in 24 hours. Given that routine testing of patients is neither clinically urgent nor meets a clear public health need, this push to introduce a non-evidence based test for uncertain gains risks inefficient use of scarce resources. Its introduction also has opportunity costs for urgent patient care and limited laboratory resources and time.

NHS England and NHS Improvement state that patient consent should be documented in the patient notes. Patient consent should always be obtained, but the need for explicitly documented consent in this way is not consistent with routine antibody testing for other acute viral infections and reflects the uncertain utility and performance of the test. Hard pressed general practitioners are being expected to provide the phlebotomy service and patient counselling. Given the uncertainties around this test, both the pre-test counselling and discussion of results are likely to be difficult and time consuming.

\section{Conclusion}

Monitoring the covid-19 epidemic is important. The only current justification for large scale SARS-CoV-2 
IgG antibody testing is for research purposes, including public health surveillance to inform epidemiology. This should be done through carefully designed studies with clear objectives, sampling frames, inclusion criteria, and consent procedures. Without this framework, it will be difficult to interpret the results of ad hoc patient testing, and their applicability will be uncertain.

The Royal College of Pathologists has set out seven principles for testing. ${ }^{3}$ Drawing on these, and on sound principles for testing of healthy asymptomatic people, ${ }^{4}$ we would like to see a carefully developed and clearly articulated strategy for serological testing, with clear scientific or clinical aims (or both) as part of a unified covid-19 response strategy with coordination across NHS England and NHS Improvement, Public Health England, and the Scientific Advisory Group for Emergencies.

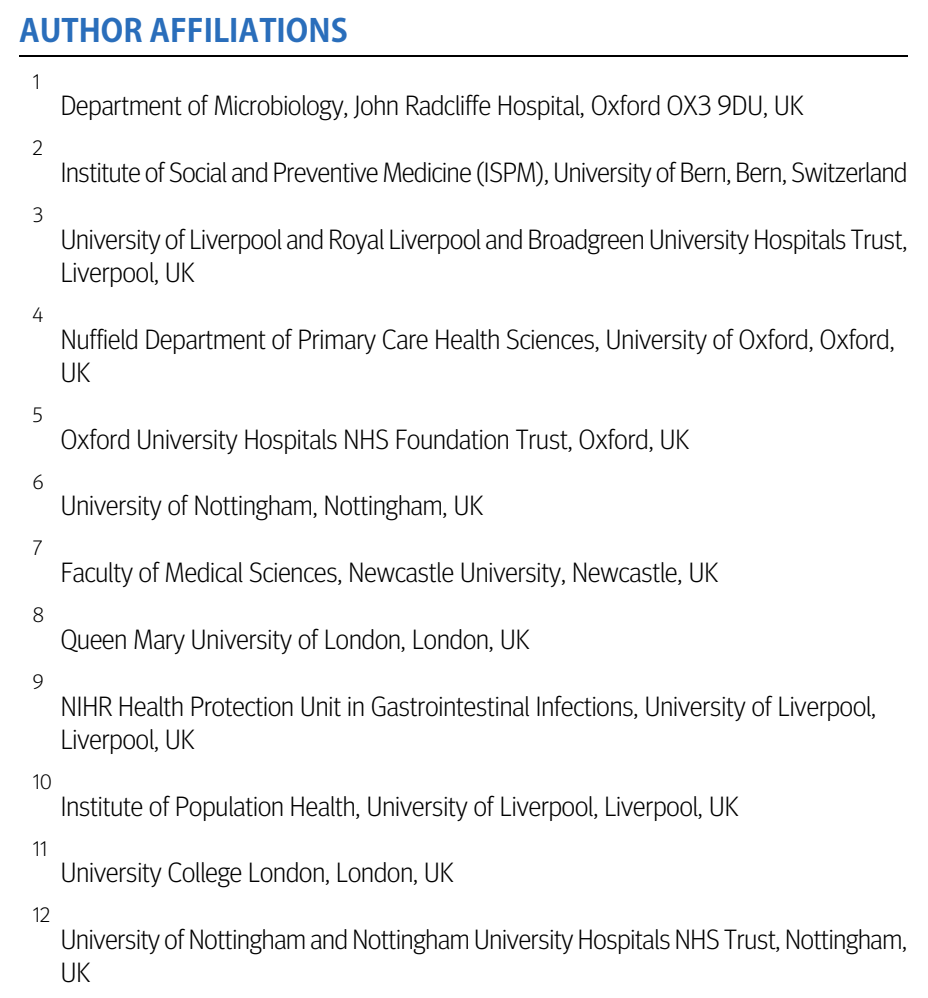

Competing interests: IB: chief data scientist adviser for AstraZeneca through the University of Liverpool. WI: speaker and consultancy fees from Roche, Janssen Cilag, Gilead Sciences, and Novartis; educational grants from Boehringer Ingelheim, Merck Sharp and Dohme, and Gilead Sciences; and research grant support from GlaxoSmithKline, Pfizer, Gilead Sciences, Janssen Cilag, Abbvie, and Bristol-Myers Squibb. All other authors: none declared.

1 Philip P, Powis S. Antibody testing programme roll out for NHS staff and patients. 25 May 2020 https://www.england.nhs.uk/coronavirus/wp-content/uploads/sites/52/2020/05/antibody-testingprogramme-letter-25-may-2020.pdf

2 Salisbury H. Helen Salisbury: Testing times for GPs. BMJ 2020;369:m2180. doi: 10.1136/bmj.m2180 pmid: 32487711

3 Royal College of Pathologists. Covid-19 testing: a national strategy. June 2020. https://www.rcpath.org/profession/on-the-agenda/covid-19-testing-a-national-strategy.html

4 Raffle AE, Mackie A, Gray JAM. Screening; Evidence and Practice. 2nd ed. Oxford University Press, 2019doi: 10.1093/med/9780198805984.001.0001. 